\title{
EL GRADO DE ALFABETIZACIÓN EN SALUD Y ADHERENCIA AL TRATAMIENTO EN PACIENTES CON HIPERTENSIÓN ARTERIAL EN UN HOSPITAL NACIONAL DE LIMA, PERÚ
}

\author{
Giovanna Rosas-Chavez ${ }^{1, a}$, Carlos A. Romero-Visurraga ${ }^{1, a}$, Elsa Ramirez-Guardia',a, Germán Málaga ${ }^{2, b}$
}

\begin{abstract}
RESUMEN
Objetivos. Evaluar el nivel de alfabetización en salud y el grado de adherencia al tratamiento en pacientes hipertensos que acuden al consultorio externo en un hospital de Lima, Perú. Materiales y métodos. Se realizó un estudio transversal en 276 pacientes, quienes llenaron un cuestionario con características generales, el test de SAHLSA-50 y el test de Morisky-Green. Se utilizó la prueba de Chi Cuadrado para evaluar la asociación entre las variables independientes con la adherencia al tratamiento y con la alfabetización en salud. Resultados. Se encontró un $36 \%$ de inadecuada alfabetización sanitaria en salud y un $15 \%$ de adherencia al tratamiento. No se halló asociación entre estas variables $(p=0,155)$. Hubo asociación entre ser jefe de familia $(p=0,033)$ y tener pareja $(p=0,044)$ con el grado de alfabetización en salud. Conclusiones. El porcentaje de alfabetización en salud fue similar al de estudios peruanos y latinoamericanos, y el grado de adherencia al tratamiento fue una de las más bajas reportadas. No se encontró asociación significativa entre éstas dos variables.
\end{abstract}

Palabras clave: Adherencia al tratamiento; Alfabetización en salud; Hipertensión (fuente: DeCS BIREME).

\section{THE DEGREE OF HEALTH LITERACY AND TREATMENT COMPLIANCE IN PATIENTS WITH HIGH BLOOD PRESSURE IN A NATIONAL HOSPITAL IN LIMA, PERU}

\begin{abstract}
Objectives. To assess the level of health literacy and treatment compliance in high-blood pressure patients attending an outpatient clinic in a hospital in Lima, Peru. Materials and Methods. A cross-sectional study was conducted in 276 patients, who filled out a questionnaire with general characteristics, the SAHLSA-50 Test, and the Morisky-Green Test. The Chi-square test was used to assess the association between independent variables with treatment compliance and health literacy. Results. We found a $36 \%$ of inadequate health literacy and $15 \%$ treatment compliance. No association was found between these variables $(p=0.155)$. There was an association between being a head of household $(p=0.033)$ and having a partner $(p=0.044)$ with the degree of health literacy. Conclusions. The percentage of health literacy was similar to that of Peruvian and Latin American studies, and the degree of treatment compliance was one of the lowest reported. No significant association was found between these two variables.
\end{abstract}

Keywords: treatment compliance, health literacy, hypertension (source: DeCS BIREME).

\section{INTRODUCCIÓN}

La alfabetización en salud (AS) es «el grado con el que los individuos pueden entender, aplicar y utilizar la información brindada por el personal de salud para tomar decisiones respecto al cuidado de sus enfermedades y promoción de su salud con el objetivo de mejorar su calidad de vida» (1). Se ha descrito que las personas de mayor edad dependientes de ayuda económica gubernamental (2), de estratos socioeconómicos bajos con bajo nivel educacional ${ }^{(3)}$, pertenecientes a minorías étnicas y migrantes recientes ${ }^{(2)}$ son los grupos de personas con mayor vulnerabilidad a una inadecuada alfabetización en salud.

\footnotetext{
Escuela de Medicina, Universidad Peruana Cayetano Heredia. Lima, Perú.

Servicio de Medicina Interna, Hospital Cayetano Heredia. Lima, Perú

Médico cirujano; ${ }^{\mathrm{b}}$ maestría en Medicina

Recibido: 11/02/2019 Aprobado: 08/05/2019 En línea: 28/06/2019
}

Citar como: Rosas-Chavez G, Romero-Visurraga CA, Ramirez-Guardia E, Málaga G. El grado de alfabetización en salud y adherencia al tratamiento en pacientes con hipertensión arterial en un hospital nacional de Lima, Perú. Rev Peru Med Exp Salud Publica. 2019;36(2):214-21. doi: http://dx.doi.org/10.17843/rpmesp.2019.362.4279. 
En países europeos, la inadecuada alfabetización en salud oscila entre el $2 \%$ y $27 \%$, siendo Austria y Bulgaria los países con las menores tasas ${ }^{(3)}$. En Estados Unidos, se reportó que el $36 \%$ de su población presenta una inadecuada alfabetización en salud, lo que se manifiesta por la falta de entendimiento de las enfermedades y por el desconocimiento de los controles que deben seguir ${ }^{(4)}$.

En Latinoamérica, pocos estudios han descrito el grado de alfabetización en salud. En Brasil, la prevalencia estimada de una inadecuada alfabetización en salud fue de $32 \%{ }^{(5)}$. En Chile, un estudio reportó un grado de alfabetización de $36 \%$, siendo el grado de instrucción, una de las principales variables relacionadas ${ }^{(6)}$. En Argentina, se reportó una prevalencia de $30 \%$, siendo las personas con una instrucción formal menor de siete años quienes presentaban mayor riesgo ${ }^{(7)}$. En Perú, el grado de una inadecuada alfabetización en salud se ha descrito entre $34 \%$ y $43 \%$ en hospitales públicos, siendo la edad del paciente, el grado de instrucción, el desempleo y la afiliación al seguro integral de salud (SIS) los factores que influyen en la misma ${ }^{(8,9)}$. Además, aquellos pacientes con una inadecuada alfabetización en salud presentan mayores ingresos a los servicios de emergencias y hospitalización, y se ha observado mayor mortalidad en adultos mayores ${ }^{(10)}$.

El impacto de una inadecuada alfabetización en salud en la no adherencia al tratamiento se ve expresada de diferentes maneras, como el de no tener la capacidad de lidiar con enfermedades crónicas ${ }^{(11)}$, no acudir a las citas médica, no seguir el tratamiento de la forma que el médico recomienda, firmar documentos sin entender los riesgos que esto conlleva o, no firmarlos por no comprender los beneficios del tratamiento ${ }^{(12)}$

Un metaanálisis, examinó la relación entre alfabetización en salud y la adherencia al tratamiento en pacientes con enfermedades cardiovasculares, encontrando una asociación positiva entre estas dos variables ${ }^{(13)}$. Asimismo, en una población de adultos mayores con enfermedades crónicas en Corea, se encontró que una adecuada alfabetización está asociada a una mayor adherencia al tratamiento ${ }^{(14)}$. En contraste, en una población de pacientes con artritis reumatoide, se reportó una adecuada alfabetización en salud $(93 \%)$ y gran adherencia al tratamiento (84\%), sin embargo, no se encontró asociación entre estas dos variables ${ }^{(15)}$.

Por lo expuesto, se puede hipotetizar que una adecuada alfabetización en salud pudiera generar una mejor adherencia al tratamiento en una enfermedad crónica como la HTA, sin embargo, esta asociación no ha sido evaluada en nuestro medio. Por lo tanto, este estudio tiene como objetivo evaluar el nivel de alfabetización en salud, así como el grado de adherencia al tratamiento de una población de

\section{MENSAJES CLAVE}

Motivación para realizar el estudio. La adherencia al tratamiento en enfermedades crónicas como hipertensión arterial (HTA) es muy importante para prevenir complicaciones. En Perú, menos de la mitad de personas con HTA cumplen su tratamiento. Creemos que la alfabetización en salud influye en este problema.

Principales hallazgos. El 35\% de los pacientes presentó inadecuada alfabetización en salud y sólo el $15 \%$ presentó adherencia al tratamiento antihipertensivo. No se encontró asociación entre ambas variables.

Implicancias. Se deben establecer programas que mejoren aspectos de cuidado y educación en salud, y así promover el empoderamiento, autocuidado y la toma de decisiones compartidas.

pacientes con HTA que acuden a un consultorio externo en un hospital de Lima, Perú.

\section{MATERIALES Y MÉTODOS}

\section{DISEÑO DE ESTUDIO}

Estudio transversal realizado en pacientes que fueron atendidos en el Hospital Cayetano Heredia entre agosto del 2016 y marzo del 2017.

\section{POBLACIÓN DE ESTUDIO}

El presente estudio, incluyó a pacientes que cumplían con los siguientes criterios: manifestar su deseo de participar voluntariamente en el estudio, ser mayor de 18 años, ser portador de HTA y acudir a la consulta externa de los consultorios de medicina o cardiología. Se excluyó a quienes no desearon participar en el estudio, no hablaban español o tuvieran una discapacidad que impidiera entender los cuestionarios.

\section{VARIABLES}

Se recolectaron variables sociodemográficas como edad, sexo, presencia de pareja, estado como jefe de familia, presencia de cuidador (persona diferente a la pareja que se encarga del cuidado del paciente), financiamiento de enfermedad, grado de instrucción, así como la presencia de comorbilidades, tiempo de enfermedad relacionada a HTA y afiliación al SIS.

\section{MUESTRA}

El tamaño de muestra fue estimado teniendo en cuenta una prevalencia de una adecuada alfabetización en salud de alrededor de $55 \%{ }^{(9)}$, una adherencia al tratamiento de HTA de $42 \%{ }^{(16)}$ y un poder estadístico de $95 \%$; con ello, se obtuvo una muestra mínima de 251 participantes. Se 
agregó un $15 \%$ por pérdidas esperadas, obteniendo un total de 296 participantes; sin embargo, se incluyeron 276 pacientes.

\section{INSTRUMENTOS}

Cada participante recibió un cuestionario que constaba de tres partes: a) Datos sociodemográficos, que incluían las variables edad, sexo, afiliación al SIS, presencia de comorbilidades, grado de instrucción, estado civil, posición en el grupo familiar, financiamiento de su enfermedad y tiempo de enfermedad.

b) La encuesta de alfabetización en salud (SAHLSA-50, Short Assessment of Health Literacy for Spanish Adults), que contiene 50 items, diseñada para evaluar la capacidad de un adulto de leer y entender términos médicos comunes ${ }^{(17)}$. Esta prueba consiste en leer adecuadamente la palabra índice y relacionarla correctamente a alguna de dos alternativas, contándose como una respuesta válida la correcta pronunciación y relación de la palabra. Un paciente con una adecuada AS se definió como aquel que obtuvo más de 37 respuestas correctas ${ }^{(18)}$ (Anexo 1 ).

c) La encuesta sobre adherencia al tratamiento (MoriskyGreen), que consta de cuatro preguntas acerca de la toma de medicación para el tratamiento de la enfermedad del paciente. Se consideró un paciente como adherente a su tratamiento si respondía las cuatro preguntas de la prueba el siguiente orden: NO-SI-NO-NO ${ }^{(19)}$ (Anexo 2).

Se solicitó a cada paciente que respondiera únicamente con relación al tratamiento antihipertensivo. El tiempo promedio para el llenado del cuestionario fue aproximadamente de 15 a 20 minutos.

\section{RECOLECCIÓN DE INFORMACIÓN}

Se realizó un muestreo por conveniencia. Se informó a los participantes que los cuestionarios eran anónimos y que la información sólo iba a ser utilizada para fines de la investigación. Se acompañó y explicó a cada uno de ellos cómo llenar los cuestionarios, los cuales se entregaron después de que los participantes brindaron su consentimiento informado por escrito.

\section{ANÁLISIS ESTADÍSTICO}

El análisis descriptivo se llevó a cabo mediante tablas de distribución de frecuencias. La variable edad fue presentada en tres categorías (18-29, 30-59 y >60 años). Asimismo, el tiempo de enfermedad desde el diagnóstico se dividió en intervalos de 5 años. Se utilizó el paquete estadístico STATA 15.0 (StataCorp, College Station, Texas, EE. UU.). Se consideró un valor de $p<0,05$ como estadísticamente significativo. Se evaluó la asociación entre la alfabetización en salud con la adherencia al tratamiento y con las variables independientes: sexo, grado de instrucción, presencia de pareja, existencia de comorbilidades, afiliación al SIS, tiempo de enfermedad y responsable de financiar su enfermedad, a través la prueba de Chi cuadrado de Pearson.

\section{CONSIDERACIONES ÉTICAS}

El estudio fue aprobado por la Dirección Universitaria de Investigación, Ciencia y Tecnología de la Universidad Peruana Cayetano Heredia y por el Comité Institucional de Ética en Investigación del Hospital Cayetano Heredia. Los datos obtenidos fueron confidenciales y sólo fueron revisados por los autores del estudio.

\section{RESULTADOS}

Se encuestó a 276 pacientes, encontrándose que el $67 \%$ (184) eran mujeres y que el 69\% (191) tenían 60 años o más. Con respecto al grado de instrucción, el 48,2\% tenían secundaria completa y el $42,0 \%$ tenían primaria completa. En cuanto a los aspectos sociales: el $50 \%$ (138) de los pacientes eran jefes de familia, el $57 \%$ (156) tenían pareja y el $61 \%$ (168). Asimismo, el $87 \%$ (241) de los pacientes presentaban comorbilidades.

Respecto a la financiación de la enfermedad, el $89 \%$ (245) de los pacientes contaban con SIS y un $46 \%$ (128) de los pacientes financiaban el costo de su enfermedad (gastos no cubiertos por el SIS). Finalmente, el 31,5\% (87) tenían entre uno a cinco años de enfermedad y el $22,4 \%$ (62) tenían entre cinco a diez años de enfermedad. Las características generales y sociales de la población se encuentran resumidas en la Tabla 1.

Se encontró que el 35,9\% (99) de los pacientes presentó una inadecuada alfabetización en salud y que la adherencia al tratamiento antihipertensivo en los pacientes que conformaron la muestra fue del 15,2\% (42) (Tabla 1).

No se encontró asociación estadística entre adherencia al tratamiento y la alfabetización en salud $(p=0,155)$ (Tabla 2). Asimismo, esta asociación tampoco fue significativa en los rangos de 18 a 59 años, ni en mayores de 60 años ( $p=0,073$ y $p=0,174$, respectivamente).

Por otro lado, se halló ser jefe de familia y tener pareja se asoció significativamente con el grado de alfabetización en salud ( $p=0,033$ y $p=0,044$, respectivamente) (Tabla 3). Finalmente, no se encontró asociación estadística entre las variables independientes y la adherencia al tratamiento antihipertensivo (Tabla 4). 
Tabla 1. Características generales de la muestra de pacientes atendidos en los consultorios de Medicina y Cardiología del Hospital Cayetano Heredia de Lima, Perú

\begin{tabular}{|c|c|c|}
\hline Variables & $\mathbf{n}$ & $\%$ \\
\hline \multicolumn{3}{|l|}{ Edad (años) } \\
\hline $18-29$ & 3 & 1,1 \\
\hline $30-59$ & 82 & 29,7 \\
\hline$\geq 60$ & 191 & 69,2 \\
\hline \multicolumn{3}{|l|}{ Sexo } \\
\hline Femenino & 184 & 66,7 \\
\hline Masculino & 92 & 33,3 \\
\hline \multicolumn{3}{|l|}{ Grado de Instrucción } \\
\hline Primaria completa & 116 & 42,0 \\
\hline Primaria incompleta & 4 & 1,5 \\
\hline Secundaria incompleta & 2 & 0,7 \\
\hline Secundaria completa & 133 & 48,2 \\
\hline Superior & 21 & 7,6 \\
\hline \multicolumn{3}{|l|}{ Jefe de familia } \\
\hline No & 138 & 50,0 \\
\hline Sí & 138 & 50,0 \\
\hline \multicolumn{3}{|l|}{ Pareja } \\
\hline No & 120 & 43,5 \\
\hline $\mathrm{Si}$ & 156 & 56,5 \\
\hline \multicolumn{3}{|l|}{ Cuidador } \\
\hline No & 108 & 39,1 \\
\hline $\mathrm{Si}$ & 168 & 60,9 \\
\hline \multicolumn{3}{|l|}{ Comorbilidades } \\
\hline No & 35 & 12,7 \\
\hline $\mathrm{Si}$ & 241 & 87,3 \\
\hline \multicolumn{3}{|l|}{ SIS } \\
\hline No & 31 & 11,2 \\
\hline $\mathrm{Si}$ & 245 & 88,8 \\
\hline \multicolumn{3}{|l|}{ Financiador } \\
\hline Hijos & 22 & 7,9 \\
\hline Padres & 3 & 1,1 \\
\hline Pareja & 45 & 16,3 \\
\hline Paciente & 128 & 46,2 \\
\hline Otros & 79 & 28,5 \\
\hline \multicolumn{3}{|c|}{ Tiempo de enfermedad (años) } \\
\hline$<1$ & 14 & 5,0 \\
\hline $1-4$ & 87 & 31,5 \\
\hline $5-9$ & 62 & 22,5 \\
\hline $10-14$ & 45 & 16,3 \\
\hline$>15$ & 68 & 24,6 \\
\hline \multicolumn{3}{|l|}{ Alfabetización en salud } \\
\hline Inadecuada & 99 & 35,9 \\
\hline Adecuada & 177 & 64,1 \\
\hline \multicolumn{3}{|l|}{ Adherencia al tratamiento } \\
\hline Adherente & 42 & 15,2 \\
\hline No adherente & 234 & 84,8 \\
\hline
\end{tabular}

Tabla 2. Asociación entre la alfabetización en salud y la adherencia al tratamiento en pacientes atendidos en los consultorios de medicina y cardiología del Hospital Cayetano Heredia de Lima, Perú

\begin{tabular}{lccc}
\hline $\begin{array}{l}\text { Alfabetización } \\
\text { en Salud }\end{array}$ & \multicolumn{2}{c}{ Adherencia al tratamiento } & \multirow{2}{*}{ Total } \\
\cline { 2 - 3 } & Adherente & No adherente & \\
\hline Inadecuada & 11 & 88 & 99 \\
Adecuada & 31 & 146 & 177 \\
\hline Total & 42 & 234 & 276 \\
\hline
\end{tabular}

Chi cuadrado de Pearson=2,017, valor de $p=0,155$

\section{DISCUSIÓN}

Se encontró que el $36 \%$ de pacientes atendidos con diagnóstico de HTA en los consultorios de medicina y cardiología tienen inadecuada alfabetización en salud. Este resultado concuerda con lo reportado en dos estudios realizados en hospitales de Perú, donde se encontró una inadecuada alfabetización de $34 \%$ y de $43 \%$, respectivamente ${ }^{(8,9)}$. Asimismo, esta cifra es similar a lo descrito en otros estudios realizados en América Latina ${ }^{(5,6)}$.

La adherencia al tratamiento fue del $15 \%$, cifra muy inferior a lo reportado en estudios realizados anteriormente en el mismo hospital, cuyo valor osciló entre el $37 \%$ a $41 \%{ }^{(16,20)}$. Particularmente, con el estudio de Carhuallanqui et al. quienes reportaron un $37 \%$ de adherencia utilizando el mismo instrumento (20). Se pueden esbozar una serie de hipótesis ante este hallazgo considerando que la mayoría de pacientes contaban con SIS, quizás factores como la mala atención percibida en los hospitales públicos o la desconfianza con la prestación brindada influyeron en la poca adherencia reportada, lo cual podría deteriorar la continuidad del tratamiento ${ }^{(1,21)}$. Sin embargo, dada la importancia que tiene la adherencia en el tratamiento de enfermedades crónicas como la HTA, cuyo control se propugna cada vez más estrecho por las connotaciones que tiene, no sólo sobre la enfermedad cardiovascular, sino sobre la cognición (22). El descenso en la adherencia al tratamiento debería ser motivo de preocupación, verificación y análisis.

Por otro lado, en el estudio no se halló asociación entre grado de alfabetización en salud y la adherencia al tratamiento. Diversos estudios describen que la adherencia al tratamiento está asociada a la alfabetización en salud, en especial en enfermedades cardiovasculares ${ }^{(23)}$. Esto significaría que, al mejorar la alfabetización en salud los pacientes lograrían comprender mejor la prescripción médica y estarían más dispuestos a cambiar sus estilos de vida ${ }^{(14,24)}$. Asimismo, se describe que la alfabetización en salud sería un fuerte predictor de adherencia al tratamiento en personas adultas mayores con enfermedades crónicas, ya que empodera a los pacientes a tomar mejores decisiones sobre su salud y evita complicaciones relacionadas con sus 
Tabla 3. Asociación entre variables independientes y la alfabetización en salud en pacientes atendidos en los consultorios de medicina y cardiología del Hospital Cayetano Heredia de Lima, Perú

\begin{tabular}{|c|c|c|c|c|c|}
\hline \multirow{3}{*}{ Variables } & \multicolumn{4}{|c|}{ Alfabetización en salud } & \multirow{3}{*}{ Valor de $\mathrm{p}^{*}$} \\
\hline & \multicolumn{2}{|c|}{ Inadecuada $(n=99)$} & \multicolumn{2}{|c|}{ Adecuada $(n=177)$} & \\
\hline & $\mathbf{n}$ & $\%$ & $\mathbf{n}$ & $\%$ & \\
\hline Edad (años) & & & & & 0,45 \\
\hline $18-29$ & 2 & 66,7 & 1 & 33,3 & \\
\hline $30-59$ & 27 & 32,9 & 55 & 67,1 & \\
\hline$\geq 60$ & 70 & 36,6 & 121 & 63,4 & \\
\hline Sexo & & & & & 0,594 \\
\hline Femenino & 64 & 34,8 & 120 & 65,2 & \\
\hline Masculino & 35 & 38,0 & 57 & 62,0 & \\
\hline Grado de instrucción & & & & & 0,80 \\
\hline Primaria completa & 44 & 37,9 & 72 & 62,1 & \\
\hline Primaria incompleta & 2 & 50,0 & 2 & 50,0 & \\
\hline Secundaria incompleta & 47 & 35,3 & 86 & 64,7 & \\
\hline Secundaria completa & 0 & 0,0 & 2 & 100,0 & \\
\hline Superior incompleta & 0 & 0,0 & 2 & 100,0 & \\
\hline Técnico superior & 5 & 33,3 & 10 & 66,7 & \\
\hline Superior & 1 & 25,0 & 3 & 75,0 & \\
\hline Jefe de familia & & & & & 0,033 \\
\hline No & 58 & 42,0 & 80 & 58,0 & \\
\hline Sí & 41 & 29,7 & 97 & 70,3 & \\
\hline Pareja & & & & & 0,044 \\
\hline No & 51 & 42,5 & 69 & 57,5 & \\
\hline $\mathrm{Si}$ & 48 & 30,8 & 108 & 69,2 & \\
\hline Cuidador & & & & & 0,746 \\
\hline No & 40 & 37,0 & 68 & 63,0 & \\
\hline $\mathrm{Si}$ & 59 & 35,1 & 109 & 64,9 & \\
\hline Comorbilidades & & & & & 0,18 \\
\hline No & 9 & 25,7 & 26 & 74,3 & \\
\hline $\mathrm{Si}$ & 90 & 37,3 & 151 & 62,7 & \\
\hline SIS & & & & & 0,40 \\
\hline No & 9 & 29,0 & 22 & 71,0 & \\
\hline $\mathrm{Si}$ & 90 & 36,7 & 155 & 63,3 & \\
\hline Tiempo de enfermedad (años) & & & & & 0,157 \\
\hline$<1$ & 3 & 21,4 & 11 & 78,6 & \\
\hline $1-4$ & 30 & 34,5 & 57 & 65,5 & \\
\hline $5-9$ & 17 & 27,4 & 45 & 72,6 & \\
\hline $10-14$ & 23 & 51,1 & 22 & 48,9 & \\
\hline $15-20$ & 7 & 38,9 & 11 & 61,1 & \\
\hline$>20$ & 19 & 38,0 & 31 & 62,0 & \\
\hline Financiador & & & & & 0,158 \\
\hline Hijos & 11 & 50,0 & 11 & 50,0 & \\
\hline Padres & 0 & 0,0 & 3 & 100,0 & \\
\hline Pareja & 16 & 35,6 & 29 & 64,4 & \\
\hline Paciente & 39 & 30,5 & 89 & 69,5 & \\
\hline Otros & 33 & 42,3 & 45 & 57,7 & \\
\hline
\end{tabular}

SIS: Seguro Integral de Salud

* Prueba: Chi cuadrado de Pearson

enfermedades ${ }^{25)}$. Sin embargo, en este estudio no se encontró asociación entre éstas dos variables, destacando que en el estudio citado se describe una tasa global de adherencia mayor a la que nosotros reportamos, lo cual podría ser un factor que explique estas diferencias. Esto debe incentivar a que se planteen estudios que confirmen esta información y que exploren otras asociaciones que influyan en la adherencia al tratamiento ${ }^{(14)} \mathrm{y}$ de manera 
Tabla 4. Asociación entre variables independientes y adherencia al tratamiento en pacientes atendidos en los consultorios de medicina y cardiología del Hospital Cayetano Heredia de Lima, Perú

\begin{tabular}{|c|c|c|c|c|c|}
\hline \multirow{3}{*}{ Variables } & \multicolumn{4}{|c|}{ Adherencia al tratamiento } & \multirow{3}{*}{ Valor de $\mathrm{p}^{*}$} \\
\hline & \multicolumn{2}{|c|}{ Adherente $(n=42)$} & \multicolumn{2}{|c|}{ No Adherente $(n=234)$} & \\
\hline & $\mathbf{n}$ & $\%$ & $\mathbf{n}$ & $\%$ & \\
\hline Edad (años) & & & & & 0,181 \\
\hline $18-29$ & 0 & 0,0 & 3 & 100,0 & \\
\hline $30-59$ & 8 & 9,8 & 74 & 90,2 & \\
\hline$>60$ & 34 & 17,8 & 157 & 82,2 & \\
\hline Sexo & & & & & 1,000 \\
\hline Femenino & 28 & 15,2 & 156 & 84,8 & \\
\hline Masculino & 14 & 15,2 & 78 & 84,8 & \\
\hline Grado de Instrucción & & & & & 0,515 \\
\hline Primaria completa & 17 & 14,7 & 99 & 85,3 & \\
\hline Primaria incompleta & 0 & 0,0 & 4 & 100,0 & \\
\hline Secundaria incompleta & 1 & 33,3 & 2 & 66,7 & \\
\hline Secundaria completa & 20 & 15,0 & 113 & 85,0 & \\
\hline Superior incompleta & 0 & 0,0 & 2 & 100,0 & \\
\hline Técnico superior & 4 & 26,7 & 11 & 73,3 & \\
\hline Superior & 0 & 0,0 & 4 & 100,0 & \\
\hline Jefe de familia & & & & & 0,738 \\
\hline No & 22 & 15,9 & 116 & 84,1 & \\
\hline $\mathrm{Si}$ & 20 & 14,5 & 118 & 85,5 & \\
\hline Pareja & & & & & 0,206 \\
\hline No & 22 & 18,3 & 98 & 81,7 & \\
\hline $\mathrm{Si}$ & 20 & 12,8 & 136 & 87,2 & \\
\hline Cuidador & & & & & 0,403 \\
\hline No & 14 & 13,0 & 94 & 87,0 & \\
\hline $\mathrm{Si}$ & 28 & 16,7 & 140 & 83,3 & \\
\hline Comorbilidades & & & & & 0,399 \\
\hline No & 7 & 20,0 & 28 & 80,0 & \\
\hline $\mathrm{Si}$ & 35 & 14,5 & 206 & 85,5 & \\
\hline SIS & & & & & 0,362 \\
\hline No & 3 & 9,7 & 28 & 90,3 & \\
\hline $\mathrm{Si}$ & 39 & 15,9 & 206 & 84,1 & \\
\hline Tiempo de Enfermedad (años) & & & & & 0,64 \\
\hline$<1$ & 3 & 21,4 & 11 & 78,6 & \\
\hline $1-4$ & 14 & 16,1 & 73 & 83,9 & \\
\hline $5-9$ & 11 & 17,7 & 51 & 82,3 & \\
\hline $10-14$ & 8 & 17,8 & 37 & 82,2 & \\
\hline $15-20$ & 1 & 5,6 & 17 & 94,4 & \\
\hline$>20$ & 5 & 10,0 & 45 & 90,0 & \\
\hline Financiador & & & & & 0,317 \\
\hline Hijos & 3 & 13,6 & 19 & 86,4 & \\
\hline Padres & 0 & 0,0 & 3 & 100,0 & \\
\hline Pareja & 4 & 8,9 & 41 & 91,1 & \\
\hline Paciente & 18 & 14,1 & 110 & 85,9 & \\
\hline Otros & 17 & 21,8 & 61 & 78,2 & \\
\hline
\end{tabular}

SIS: Seguro Integral de Salud

* Prueba: Chi cuadrado de Pearson 
especial, estudios cualitativos, que permitan entender el cambio en la adherencia. Otro factor que podría contribuir al bajo grado de alfabetización en salud es el deficiente rol del personal de salud en incrementar el conocimiento sanitario en los pacientes, lo que se refleja en el escaso tiempo para la atención médica. Por ello, sería interesante desarrollar estudios que busquen incrementar el grado de alfabetización sanitaria en la práctica clínica y fomentar el desarrollo de herramientas en Internet para el uso del público en general ${ }^{(26)}$.

No se encontró asociación estadística entre el grado de adherencia al tratamiento y las variables independientes estudiadas. De éstas, la literatura describe que la edad, el tener acceso a algún tipo de seguro y tener un mayor grado de instrucción están asociadas a una mayor adherencia al tratamiento ${ }^{(27)}$. Como se mencionó previamente, la población en el presente estudio tenía un menor grado de instrucción comparado con otros estudios locales ${ }^{\left({ }^{8}\right)}$, lo que posiblemente habría afectado la adherencia al tratamiento. En cuanto a la edad, los estudios que describen su relación con la adherencia al tratamiento no son concluyentes ${ }^{(27)}$. Adicionalmente, se menciona que el tener acceso a un seguro podría ser un factor asociado a mayor adherencia, esto debido a un mayor acceso a medicamentos y seguimiento de la enfermedad; en el presente estudio no se encontró asociación entre estar afiliado al SIS y la adherencia al tratamiento ${ }^{(26)}$.

Finalmente, las variables que presentaron asociación significativa a la alfabetización en salud fueron «ser jefe de familia» y «tener pareja». Esto también es diferente a lo descrito en la literatura, donde ser «adulto mayor», «menor grado de instrucción», «tener cobertura de seguro» y «menor número de veces que acuden a consulta» se describen como asociadas a la AS ${ }^{(8)}$. Es necesario mencionar que el grado de instrucción de los participantes es inferior a lo descrito en otros estudios en América y Europa ${ }^{(13)}$.

Si bien, este estudio se ha realizado en una sola institución y cuya generalización de resultados pudiera no ser extensiva a la sociedad, consideramos que refleja la situación de los hospitales públicos, donde las carencias en AS son sumamente influyentes para lograr comunicación, adherencia y autocuidado, y particularmente para lograr la toma de decisiones compartidas ${ }^{(24,28)}$, esto enmarcado en un mundo donde se considera, desde hace una década, que la deficiente «alfabetización digital en salud», sería una forma de discapacidad ${ }^{(29)}$. Es por la importancia descrita, que se propugna que la alfabetización en salud, debería ser registrada como una función vital ${ }^{(25)}$.

En este sentido y considerando una atención de calidad, en la que se controlen aspectos biológicos como la presión arterial a través de la adherencia, se vuelve necesario mejorar aspectos trascendentales de la atención sanitaria, como la alfabetización en salud. Para esto, se requiere del compromiso de todos los estamentos del sector, desde quienes toman decisiones en salud hasta quienes interactuamos con los pacientes, para lograr una práctica médica cuidadosa y empática en la que se empodere a las personas en la toma de decisiones para su mejor autocuidado.

Entre las limitaciones del estudio podemos mencionar que debido al diseño transversal no se puede establecer causalidad. La muestra no representativa y el muestreo no probabilístico podrían haber afectado la validez externa del estudio. El bajo grado de instrucción de los participantes posiblemente influyó en la asociación de esta variable con el grado de alfabetización. El autorreporte de la adherencia al tratamiento podría conllevar a sesgos, sobre todo en poblaciones con un bajo grado de escolaridad. Finalmente, no se tuvo información del control de la HTA, por lo que no se incluyó como variable independiente. Los hallazgos referidos a la alfabetización en salud son similares a otros estudios realizados con la prueba SAHLSA-50, incluso con uno validado culturalmente en Perú ${ }^{(8)}$, estudio que no estuvo disponible en el periodo de recolección de muestra, pero que no presenta diferencias en cuanto a su entendimiento.

Se concluye que no se encuentra asociación estadística entre la inadecuada alfabetización en salud y la adherencia al tratamiento. El porcentaje de adherencia al tratamiento resultó ser inferior a lo reportado en otros estudios realizados en Perú.

Contribuciones de autoría: GRC, CRV, ERG participaron en la concepción y diseño del artículo, recolección de resultados, análisis e interpretación de datos, redacción del artículo, revisión crítica del artículo, aprobación de la versión final. GM participó en la concepción y diseño del artículo, análisis e interpretación de datos, redacción del artículo, revisión crítica del artículo, aprobación de la versión final, asesoría técnica y estadística.

Conflictos de interés: En este trabajo no se encuentran conflictos de interés.

Financiamiento: El estudio fue autofinanciado por los autores

Material suplementario: Disponible en la versión electrónica de la RPMESP.

\section{REFERENCIAS BIBLIOGRÁFICAS}

1. Seldon CR, Zorn M, Ratzan SC, Parker RM. National Library of Medicine Current Bibliographies in Medicine: Health Literacy. Bethesda, MD: National Institutes of Health; 2000.
2. Office of Disease Prevention and Health Promotion. National Action Plan to Improve Health Literacy. Washington, DC: U.S. Department of Health and Human Services; 2010.
3. Sørensen K, Pelikan JM, Röthlin F, Ganahl K, Slonska Z, Doyle G, et al. Health literacy in Europe: comparative results of the European health literacy survey (HLS-EU). Eur J Public Health. 
2015;25(6):1053-8. doi: 10.1093/ eurpub/ckv043.

4. Organización Panamericana de la Salud. Perfil de Sistemas y Servicios de Salud Estados Unidos de America. 2002 [Internet]. Washington, D.C: OPS; 2002 (Citado 18 de abril 2016]. Disponible en: http://www.paho. org/hq/dmdocuments/2010/Perfil_ Sistema_Salud-Estados_Unidos_ America_2002.pdf.

5. Carthery-Goulart MT, Anghinah R, Areza-Fegyveres R, Bahia VS, Brucki SM, Damin A, et al. Performance of a Brazilian population on the test of functional health literacy in adults. Rev Saude Publica. 2009; 43(4):631-8.

6. Escoda Riveros J, Terrazas Martins F. La Alfabetización de la Salud en el Sistema de Salud Pública en Chile [Tesis]. Santiago: Universidad de Chile; 2008. Disponible en: http://repositorio.uchile. cl/bitstream/handle/2250/107916/ Alfabetizaci\%C3\%B3n\%20de\%20la\%20 Salud.pdf $?$ sequence $=4 \&$ isAllowed $=y$

7. Konfino J, Mejía R, Majdalani MP, Pérez-Stable EJ. Alfabetización en salud en pacientes que asisten a un hospital universitario. Medicina (B Aires). 2009;69(6):631-4.

8. Hidalgo Cruz JI, Valero Delgado VR. Alfabetización sanitaria evaluada mediante un instrumento validado y factores relacionados en pacientes hospitalizadosenlosServiciosdeMedicina Interna en un hospital nacional en LimaPerú 2018 [Tesis]. Lima: Universidad Peruana Cayetano Heredia; 2018. Disponible en: http://repositorio.upch. edu.pe/bitstream/handle/upch/1463/ Alfabetizacion_HidalgoCruz_Jimena. pdf ? sequence $=1$ \&isAllowed $=y$

9. Vílchez $\mathrm{C}$, Quiliano $\mathrm{R}$, Canales $\mathrm{R}$, Chiroque R. Factores demograficos asociados con el conocimiento sobre salud en un muestra de mujeres de la ciudad de Lima (Peru) en 2008: resultados del cuestionario SAHLSA-50. Rev Colomb Obstet Ginecol 2009; 60(3):223-9).

10. Berkman ND, Sheridan SL, Donahue KE, Halpern DJ, Crotty K. Low Health Literacy and Health Outcomes: An Updated Systematic Review. Ann Intern Med. 2011;155(2):97-107. doi: 10.7326/00034819-155-2-201107190-00005.

11. Safeer RS, Keenan J. Health literacy: the gap between physicians and patients. Am Fam Physician 2005;72:463-8.
12. Rothman RL, Yin HS, Mulvaney S, Co JP, Homer C, Lannon C. Health literacy and quality: focus on chronic illness care and patient safety. Pediatrics. 2009;124 Suppl 3:S315-26. doi: 10.1542/peds.2009$1163 \mathrm{H}$.

13. Keim-Malpass J, Doede A, Showalter SL. Does patient health literacy impact adherence to adjuvant endocrine therapy in breast cancer patients? Patient Prefer Adherence. 2018;13:47-51. doi: 10.2147/ PPA.S190249.

14. Miller TA. Health literacy and adherence to medical treatment in chronic and acute illness: A meta-analysis. Patient Educ Couns. 2016;99(7):1079-1086. doi: 10.1016/j.pec.2016.01.020.

15. Quinlan P, Price KO, Magid SK, Lyman S, Mandl LA, Stone PW. The relationship among health literacy, health knowledge, and adherence to treatment in patients with rheumatoid arthritis. HSS J. 2013;9(1):429. doi: 10.1007/s11420-012-9308-6.

16. Fernandez-Arias M, Acuna-Villaorduna A, Miranda JJ, Diez-Canseco F, Malaga G. Adherence to pharmacotherapy and medication-related beliefs in patients with hypertension in Lima, Peru. PLoS One. 2014;9(12):e112875. doi: 10.1371/ journal.pone.0112875.

17. Lee S-YD, Stucky BD, Lee JY, Rozier RG, Bender DE. Short Assessment of Health Literacy-Spanish and English: a comparable test of health literacy for Spanish and English speakers. Health Serv Res. 2010;45(4):1105-20. doi: 10.1111/j.1475-6773.2010.01119.x.

18. Lee SY, Bender DE, Ruiz RE, Cho YI. Development of an easy-to-use Spanish Health Literacy test. Health Serv Res. 2006;41:1392-412.

19. Val Jimenez A, Amoros Ballestero G, Martinez Visa P, Fernandez Ferre ML, Leon Sanroma M. Descriptive study of patient compliance in pharmacologic antihypertensive treatment and validation of the Morisky and Green test. Aten Primaria 1992;10:767-70.

20. Carhuallanqui R, Diestra-Cabrera G, Tang-Herrera J, Málaga G. Adherencia al tratamiento farmacológico en pacientes hipertensos atendidos en un hospital general. Rev Med Hered. 2010;21:197-201.

21. Gutiérrez C, Romaní Romaní F, Wong P, Del Carmen Sara J. Brecha entre cobertura poblacional y prestacional en salud: un reto para la reforma de salud en el Perú. An Fac med. 2018;79(1):65-70. doi: 10.15381/anales.v79i1.14595.
22. Jiménez-Balado J, Riba-Llena I, Abril O, Garde E, Penalba A, Ostos E, et al. Cognitive Impact of Cerebral Small Vessel Disease Changes in Patients With Hypertension. Hypertension. 2019;73(2):342-349. doi: 10.1161/ HYPERTENSIONAHA.118.12090.

23. Mayo-Gamble TL, Mouton C. Examining the Association Between Health Literacy and Medication Adherence Among Older Adults. Health Commun. 2018;33:1124-30.

24. Laspiur S. Abordaje integral de personas con enfermedades crónicas no transmisibles - modelo MAPEC. Ciudad Autónoma de Buenos Aires: Ministerio de Salud de la Nación. REDES; 2016. Disponible en: http://www.msal.gov.ar/ redes/abordaje-integral-de-personas-conenfermedades-cronicas-no-transmisibles/

25. Traver M, Basagoiti I, Martinez-Millana A, Fernandez-Llatas C, Traver V. Experiences of a general practitioner in the daily practice about Digital Health Literacy. The real needs. Conf Proc IEEE Eng Med Biol Soc 2016;2016:5644-7.

26. SawkinMT,DeppeSJ, ThelenJ,StonerSC, Dietz CA, Rasu RS. Health Literacy and Medication Adherence Among Patients Treated in a Free Health Clinic: A Pilot Study. Health Serv Res Manag Epidemiol. 2015 Jun 4;2:2333392815589094. doi: $10.1177 / 2333392815589094$.

27. Mathes T, Jaschinski T, Pieper D. Adherence influencing factors - a systematic review of systematic reviews. Arch Public Health. 2014;72(1):37. doi: 10.1186/2049-3258-72-37.

28. Pieterse AH, Stiggelbout AM, Montori VM. Shared Decision Making and the importance of Time. JAMA. 201919 de abril. Doi: 10.1001/ jama.2019.3785.

29. Norman CD, Skinner HA. eHealth Literacy: Essential Skills for Consumer Health in a Networked World. J Med Internet Res. 2006;8(2):e9. doi: 10.2196/ jmir.8.2.e9.

Correspondencia: Germán Málaga Rodriguez Dirección: Calle Las Cantutas 479, Casuarinas Sur. Santiago de Surco. Lima, Perú Teléfono: (511) 992768300 